\title{
Golgi during Development
}

\author{
Weimin Zhong \\ Department of Molecular, Cellular, and Developmental Biology, Yale University, New Haven, \\ Connecticut 06520 \\ Correspondence: weimin.zhong@yale.edu
}

The Golgi is essential for processing proteins and sorting them, as well as plasma membrane components, to their final destinations. Not surprisingly, this organelle, a major compartment of the secretory pathway, is an important venue for regulating many aspects of development in both invertebrates and vertebrates. Through its role as a site for protein cleavage and glycosylation as well as through changes in its spatial organization and secretory trafficking, the Golgi exerts highly specific effects on cellular differentiation and morphogenesis by spatially and temporally constraining developmental pathways.

\begin{abstract}
A key goal of developmental biology is to Aunderstand how cells make choices between proliferation and differentiation and how seemingly indistinguishable populations of cells eventually possess unique properties and undergo distinct morphological changes along the way. At the molecular level, proteins such as nuclear transcription factors that are expressed in a cell type-specific manner endow cells with unique morphological and functional characteristics by regulating the expression of a unique set of genes in each cell type. Interestingly, proteins that are widely expressed, including many cell-surface receptors and their ligands on neighboring cells or in the environment, also play essential roles in driving cellular differentiation. They, however, act in a more context-dependent manneroften through tightly controlled regulation of their activity spatially and temporally-to enable a wide variety of cell types to acquire unique properties.
\end{abstract}

Proteins that reach the cell surface or are secreted to the extracellular environment are processed and sorted through the secretory pathway, which can also divert proteins to distinct subcellular locations (reviewed in Lee et al. 2004). The secretory pathway comprises the endoplasmic reticulum (ER), the Golgi, and additional components of the endomembrane system including vacuoles/lysosomes and a variety of endosomal compartments. Many nascent polypeptide chains are released by ribosomes into ER lumen, where they are folded by chaperones and undergo an initial set of modifications including the addition of $\mathrm{N}$-linked glycan chains and hydroxylation of proline residues. The proteins are then transported by COPII vesicles to the Golgi, where they can undergo further modifications such as the sequential addition of more glycan side chains, which can be very elaborate, as well as proteolytic cleavage. Once properly processed, proteins are sorted by the trans-Golgi network

Editors: Graham Warren and James Rothman

Additional Perspectives on The Golgi available at www.cshperspectives.org

Copyright (C) 2011 Cold Spring Harbor Laboratory Press; all rights reserved; doi: 10.1101/cshperspect.a005363 Cite this article as Cold Spring Harb Perspect Biol 2011;3:a005363 
W. Zhong

(TGN) to either the cell surface or other endomembrane compartments.

This article is not intended as a comprehensive overview as to how the Golgi affects embryonic development through its general importance in protein processing and secretory trafficking. Instead, I shall highlight examples of how the Golgi is used as a venue for spatially and temporally constraining the reach of signaling pathways so that, despite the near ubiquitous presence of their key components, such pathways can be used reiteratively in a cell type- and developmental stage-specific manner to influence cellular differentiation. I shall also highlight how changes in Golgi organization help cells to coordinate cell-cycle progression and cell-fate specification when they divide asymmetrically or develop unique cellular domains such as neuronal axons and dendrites.

\section{CONTROL OF RECEPTOR ACTIVATION THROUGH PROTEIN CLEAVAGE IN THE GOLGI}

As proteins transit through the secretory pathway, some undergo proteolytic cleavage in the Golgi. Signaling by the epidermal growth factor (EGF) receptor (EGFR) during development in Drosophila melanogaster is an example of how the activation of a receptor can be spatially and temporally constrained by regulating the production of its active ligand as the inactive precursor transits through the ER and the Golgi after synthesis (Lee et al. 2001; Urban et al. 2001; Tsruya et al. 2002).

EGFR is an evolutionarily conserved family of transmembrane proteins that plays pleiotropic roles during vertebrate and invertebrate development and is activated by small secreted signaling molecules like EGF and transforming growth factor $\alpha$ (TGF- $\alpha$ ) (reviewed in Bogdan and Klambt 2001; Urban 2006). In Drosophila, the single EGFR (DER) and its ligand Spitz are expressed by virtually all cells and are involved in the development of virtually every tissue (Price et al. 1989; Schejter and Shilo 1989; Rutledge et al. 1992). Spitz is a member of the TGF- $\alpha$ family of transmembrane proteins with an extracellular EGF motif (Schejter and
Shilo 1989). Only the extracellular portion of Spitz, which is generated through proteolytic cleavage, can activate DER. Two other proteins, Rhomboid (Rhomboid-1, -2, and -3) and Star, regulate the cleavage of Spitz and are more restricted in their expression, which prefigures DER activation. Furthermore, Rhomboid proteins and Star are required in the signaling rather than the receiving cells (Golembo et al. 1996; Guichard et al. 1999). Studies by Freeman, Shilo, and their colleagues revealed an interesting mechanism that explains how DER activation is prefigured (Lee et al. 2001; Urban et al. 2001; Tsruya et al. 2002). As shown in Figure 1A, the Rhomboid-1 protein is confined to the Golgi (or a late compartment of the TGN), whereas Star is more widely distributed within the cell and present throughout the secretory pathway, including the ER and the Golgi (as well as the plasma membrane). Star is required to chaperone the Spitz precursor from the ER to the Golgi, where Rhomboid-1, an intramembrane serine protease, cleaves Spitz to generate the active form that is then trafficked through the secretory pathway to reach the cell surface and is secreted. In cells that do not have Star, Spitz is retained in the ER and thus cannot be cleaved into the active form as Rhomboid-1 is localized to the Golgi (Lee et al. 2001).

This mechanism - that activation of EGFR signaling requires proteolytic cleavage of the ligand in the signaling cell-explains why DER activity is restricted to domains much narrower than the expression domain of its ligand Spitz during development (Rutledge et al. 1992; Gabay et al. 1997) and why simply overexpressing the full length Spitz protein is not sufficient to activate the receptor (Schweitzer et al. 1995). EGFR signaling, for example, is used reiteratively in a temporally and spatially constrained manner to sequentially generate different cell types in the Drosophila eye (reviewed in Freeman 1996). The fly has a compound eye comprising about 750 ommatidia with the same complement of eight photoreceptors, R1 to R8, and 13 support cells (four cone cells and nine pigment cells, although three of the latter are shared with neighboring 
A

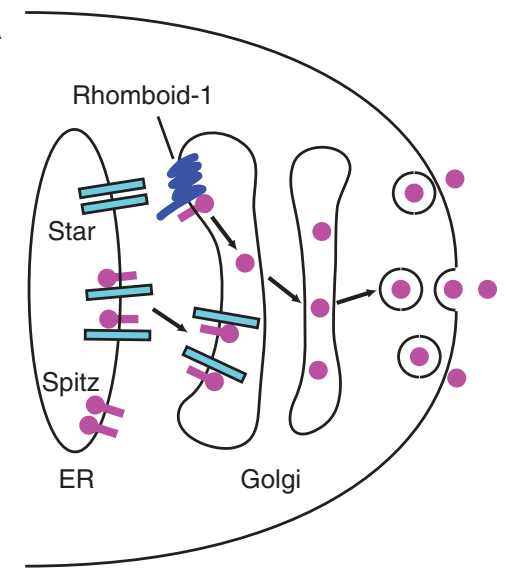

B

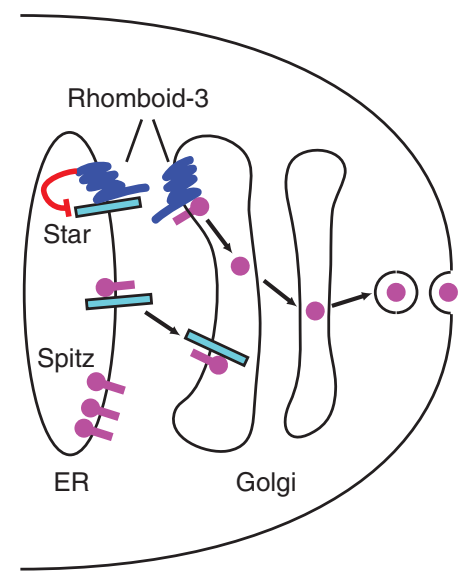

Figure 1. Regulation of Spitz production by Rhomboid proteins and Star. (A) Star chaperones the inactive Spitz precursor from the ER to the Golgi where it is cleaved by the 7-transmembrane protease Rhomboid-1, generating the active Spitz ligand that is secreted to activate EGFR. (B) Rhomboid-3 also resides in the ER where it cleaves Star to inactivate its ability to chaperone Spitz to the Golgi. Rhomboid-3 can cleave Spitz in both the ER and the Golgi to generate the active form. However, with lower levels of Star in the ER, a smaller amount of active Spitz is produced and secreted.

ommatidia). The eye develops from a monolayer epithelium, the eye imaginal disc. Cells forming imaginal discs are set aside during embryonic development, proliferate during the three larval stages, and are used to generate external adult structures like eyes, wings, and legs during pupal transformation. Differentiation of eye discs starts around the beginning of the third instar, with the appearance of a groove known as morphogenetic furrow. The furrow sweeps anteriorly across the disc, and cells posterior to the furrow differentiate to form rows of ommatidia. Within each ommatidium, photoreceptors and support cells form in an orderly fashion. The R8 photoreceptor is born first, generated through a DER-independent mechanism called lateral inhibition (see discussion below). Once the founder R8 is established, reiterative rounds of DER activation is used to sequentially generate the remaining photoreceptors (as well as cone and pigment cells). The R8 first induces two neighbors to become R2 and R5, and the three cells then induce their respective neighbors to become R3 and R4, followed by R1 and R6, and lastly R7. Early expression of Rhomboid (Rhomboid-1 and -3) and Star only occurs in R8, R2, and R5. Consequently, despite nearly universal presence of DER and Spitz, loss of either Star or Rhomboid-1 and -3 is sufficient to prevent the formation of all photoreceptors (Heberlein and Rubin 1991; Heberlein et al. 1993; Wasserman et al. 2000).

Rhomboid proteins are a family of evolutionarily conserved but functionally divergent membrane proteases, with seven rhomboid-like genes in Drosophila and at least six in vertebrates (reviewed in Freeman 2004; Urban 2006). Studies of the roles of rhomboid-like genes in Drosophila eye development have also yielded intriguing evidence that different Rhomboid proteins use the secretory pathway differently to regulate DER signaling (Tsruya et al. 2007; Yogev et al. 2008). Rhomboid-1 is expressed in many tissues and is a major regulator of EGFR signaling. It, however, is dispensable in the developing eye, where Rhomboid-3 (also known as Roughoid) is the key Rhomboid for regulating EGFR-dependent cell recruitment, although Rhomboid-1 and -3 double-mutation causes more severe defects, suggesting they act together to control EGFR signaling (Wasserman et al. 2000). Like Rhomboid-1, Rhomboid-3 can cleave the Spitz precursor to generate the 
W. Zhong

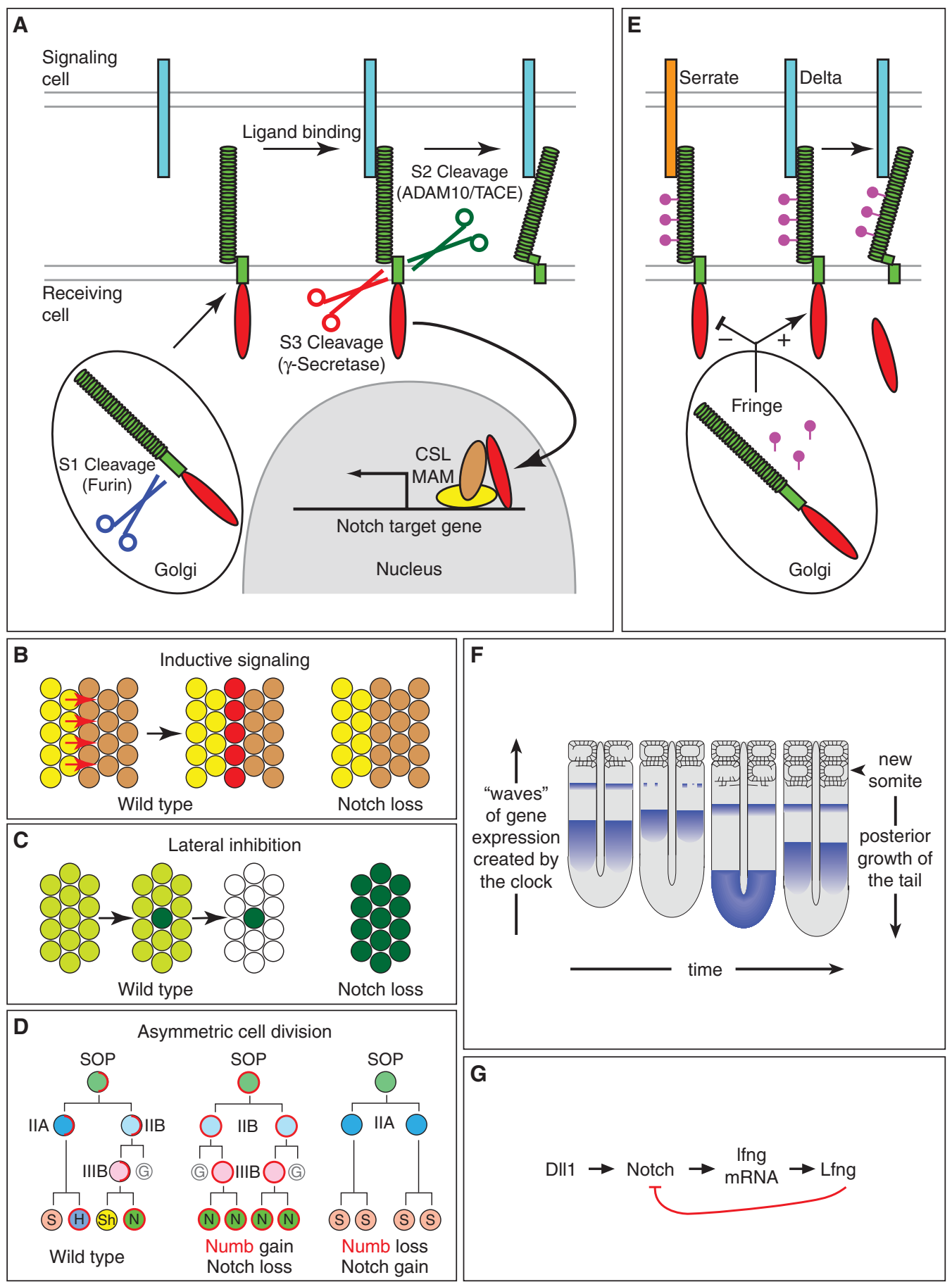

Figure 2. Regulation and the roles of Notch signaling. (A) Activation of Notch signaling requires a series of cleavage events. Full-length Notch proteins are cleaved in the Golgi by a Furin-like protease (S1 cleavage), whereas Notch activation by ligands such as Delta triggers ECD shedding by the metalloprotease ADAM10 or TACE (S2 cleavage), which in turn triggers S3 cleavage by the $\gamma$-Secretase complex to release the ICD. (See facing page for legend.) 
active ligand for DER activation. Unlike Rhomoboid-1, Rhomboid-3 is also in the ER, where it cleaves Star and inactivates its ability to chaperone Spitz to the Golgi. This dual role of Rhomboid-3 points to an interesting mechanism by which the protein activates DER signaling by generating the active ligand but also limits the range of DER activation by limiting the production of active Spitz through a reduction in Star level to retain Spitz in the ER (Fig. 1B). This tight control of EGFR signaling is essential during eye development because the pool of undifferentiated cells is limited (Tsruya et al. 2002). The genome of the flour beetle Tribolium castaneum contains a single rhomboid gene, and the protein is present in the ER and other secretory compartments. The beetle Rhomboid, however, is incapable of cleaving Star, and this apparently enables EGFR to signal over a longer range in beetle neurogenesis (Rousso et al. 2010). It is worth pointing out that mammals appear not to use Rhomboid proteins to modulate EGFR signaling, raising the possibility that different species evolve to use Rhomboid proteins differently during development (reviewed in Freeman 2004; Urban 2006).

Notch proteins, another family of transmembrane receptors, represent an example of the importance of protein cleavage in the Golgi for endowing a receptor with the capability of receiving a signal at the cell surface. Notch proteins - one in Drosophila, two in Caenorhabditis elegans (LIN-12 and GLP-1), and four in mammals (Notch1-4) — are similar in amino acid sequence and virtually identical structurally, with an extracellular domain (ECD) that mediates ligand binding, a single pass transmembrane domain (TMD), and an intracellular domain (ICD). Studies by Artavanis-Tsakonas, Israël, and colleagues showed that a Notch receptor is first cleaved ( $\mathrm{S} 1$ cleavage) in the Golgi during transport to the cell membrane by a Furin-like proteinase, and the mature receptor is a heterodimer of its ECD tethered to the TMD/ICD through noncovalent, calciumdependent interactions (Fig. 2A). This S1 cleavage is essential for producing a functional receptor (Blaumueller et al. 1997; Logeat et al. 1998; Lake et al. 2009).

\section{MODULATION OF RECEPTOR ACTIVATION THROUGH PROTEIN GLYCOSYLATION IN THE GOLGI}

Although there is no evidence that Notch S1 cleavage in the Golgi is regulated in a manner that is cell type- or developmental stagespecific, Notch signaling is an excellent example to highlight both the general importance of glycosylation and how glycosylation in the Golgi is used to provide spatial and temporal specificity in select developmental contexts (reviewed in Tien et al. 2009; Jafar-Nejad et al. 2010; Takeuchi and Haltiwanger 2010).

Notch signaling plays diverse roles in metazoans to influence a wide variety of developmental events, including cell proliferation,

Figure 2. (Continued) Freed Notch ICD enters the nucleus without a need for further Notch activation and activates target genes by cooperating with the DNA-binding protein CSL and its coactivator MAM. (B) Schematic drawings of the process of inductive signaling and the effect of Notch loss during this process. $(C)$ Schematic drawings of the process of lateral inhibition and the effect of Notch loss during this process. $(D)$ Schematic drawings of the SOP lineage that produces Drosophila ES organs and the effect of changes in Numb and Notch activity. In the wild type, Numb (in red) localizes asymmetrically in dividing SOP cells and segregates to the IIB daughter to promote its fate. Subsequently, IIA and IIIB cells asymmetrically segregate newly synthesized Numb to distinguish their daughter cells. S, socket cell; H, hair cell; Sh, sheath cell; N, neuron. The glial cell (G) is not part of the ES organ, and IIB divisions appear to be insensitive to Numb activity. (E) Fringe adds GlcNac to the $O$-fucose sites on Notch EGF repeats in the Golgi, which makes Notch more sensitive to activation by Delta but less so by Serrate during Drosophila wing margin development. (F) Schematic drawings of cyclic gene expression driven by the segmentation clock during somitogenesis in vertebrate animals (courtesy of Scott Holley, Yale University). $(G)$ Lfng provides a negative feedback loop for periodic inhibition of Notch, which in turn controls the rhythmic expression of cyclic genes in the posterior PSM in chick and mice. 
W. Zhong

differentiation, and death (reviewed in Mumm and Kopan 2000; Bray 2006; Louvi and Artavanis-Tsakonas 2006). An interesting feature of this pathway is that the receptor, through its ICD, directly acts as a regulator of gene expression in the nucleus on activation, which is accomplished through a series of cleavage events. Notch activation on ligand binding to its ECD triggers the S2 cleavage at the extracellular juxtamembrane region by a member of the ADAM family of metalloproteases, ADAM10, or tumor necrosis factor converting enzyme (TACE, also known as ADAM17). The S2 cleavage generates a substrate for S3 (and S4) cleavage in the transmembrane domain, which is catalyzed by the $\gamma$-secretase complex and releases into the nucleus the freed ICD that cooperates with the DNA-binding protein CSL, named after $\mathrm{CBF} 1, \mathrm{Su}(\mathrm{H})$, and LAG-1, and its co-activator Mastermind (MAM) (Fig. 2A). The Notch ligands are also transmembrane proteins, and this signaling pathway is used in many contexts to directly influence cell-cell interaction so that neighboring cells can choose differently among the available developmental options.

In Drosophila, Notch-mediated cell-cell interaction is used in three types of developmental contexts: lateral inhibition, asymmetric cell division, and inductive signaling (reviewed in Haines and Irvine 2003). In the developing nervous system, for example, neural precursor cells emerge from clusters of ectodermal cells that have equal developmental potential, but only a small fraction of the ectodermal cells eventually become neural precursors whereas the remaining cells choose other fates. This is accomplished through lateral inhibition, by which an emerging neural precursor cell prevents the surrounding cells from adopting the same fate (Fig. 2C). As mentioned above, R8 photoreceptors in the Drosophila eye imaginal disc are generated through lateral inhibition. Whereas R8 cells help to generate other cell types in the eye by recruiting their neighbors through EGFR signaling, most neural precursor cells divide asymmetrically to produce two different daughter cells (Fig. 2D) (discussed in more detail below). Both processes are Notch dependent; in the absence of Notch signaling, all ectodermal cells within the cluster become neural precursor cells, which then divide symmetrically to produce two daughter cells with the same fates. Drosophila wing development represents the third type of Notch-dependent cell-cell interaction, inductive signaling, that generates compartments to separate cells into populations that do not intermix (Fig. 2B). Each adult wing arises from a wing imaginal disc, which is basically a two-dimensional sheet of epithelium with a single layer of cells. It is divided into dorsal cells and ventral cells, and the two populations do not intermix, with a clearly established boundary separating them. As the wing disc folds during metamorphosis, the dorsal and ventral cells form the two opposing cell layers of the wing blade, whereas cells at the dorsal-ventral boundary form the edge of the wing (wing margin). Notch-mediated interaction between dorsal and ventral cells is essential for boundary formation and wing development.

Genetic studies of genes encoding glycosyltransferases have provided evidence of not only the importance but also the specificity of glycosylation in modulating Notch activity in different developmental contexts (reviewed in Tien et al. 2009; Jafar-Nejad et al. 2010; Takeuchi and Haltiwanger 2010). Notch proteins are widely expressed, and their activity often is not regulated at the level of gene and protein expression. The EGF-like repeats in the Notch ECD contain the sites for at least two types of $O$-linked glycosylation, $O$-fucosylation, and $O$-glucosylation. The first step in Notch glycosylation is the addition of fucose to the serine or threonine site in the ER by the enzyme O-fucosyl transferase (OFutl in Drosophila and Poftul in mammals) (reviewed in Stanley 2007). The $O$-fucose site can be elongated by adding an $N$-acetylglucosamine (GlcNAc), which occurs in the Golgi by the enzyme $\beta 1,3-N$-acetylglucosaminyltransferase encoded by evolutionarily conserved fringe genes (Fig. 2E) (Bruckner et al. 2000; Moloney et al. 2000; Munro and Freeman 2000). In Drosophila, the OFut1 gene acts cell autonomously in the signal-receiving cell and is essential for 
most, and possibly all, aspects of Notch signaling, and this requirement for Ofut1 is upstream of Notch activation (Okajima and Irvine 2002). By contrast, fringe loss affects only specific aspects of Notch signaling. Among the three aspects of Notch signaling described above, fringe loss does not appear to affect lateral inhibition and asymmetric cell division but severely disrupts boundary formation in the wing imaginal disc (Irvine and Wieschaus 1994; Kim et al. 1995; Rauskolb et al. 1999). Fringe is expressed in the dorsal but not the ventral cells, and its loss causes dorsal cells to violate the compartment boundary, whereas its ectopic expression causes ventral cells to violate the compartment boundary. Fringe establishes the compartment boundary primarily by positioning a stripe of Notch activation along the border of its expression. This is accomplished by modulating the sensitivity of the Notch receptor toward the ligands; glycosylation by Fringe potentiates Notch activation by Delta while at the same time inhibits Notch activation by Serrate in the dorsal cells (Fig. 2B) (Fleming et al. 1997; Panin et al. 1997; Klein and Arias 1998). It is worth pointing out that Serrate and Delta are functionally redundant in mediating Notch signaling between the two daughter cells during asymmetric divisions by neural precursor cells; reducing either one alone has mild or no effect, whereas removing both causes the two daughter cells to choose the same fates as they do in the absence of Notch signaling (Zeng et al. 1998).

In vertebrates, the influence of Fringe on Notch signaling is complicated by the presence of three Fringe proteins (Lunatic, Manic, and Radical) as well as multiple Notch receptors and ligands. Nevertheless, there is evidence that Fringe proteins can influence the signaling by different Notch receptor-ligand combinations (reviewed in Haines and Irvine 2003). That glycosylation in the Golgi is used to regulate subsets of Notch signaling also appears to be true. Mice mutant for Pofut1 show severe defects in somitogenesis, vasculogenesis, cardiogenesis, and neurogenesis, which resemble the phenotypes shown by mice with a complete loss of Notch signaling (Shi and Stanley 2003).
Lunatic fringe ( $L f n g$ ) mutant mice, on the other hand, show highly specific phenotypes, exemplified by defects in somitogenesis (Evrard et al. 1998; Zhang and Gridley 1998).

Somites are paired aggregates of mesodermderived cells that form along the neural tube of vertebrate embryos. Somites give rise to bones of the vertebrate column, ribs, attached skeletal and limb muscles, as well as other mesodermal tissues. The process of somitogenesis is characterized by its periodicity and directionality, with somites forming one pair at a time during embryogenesis from the unsegmented presomatic mesoderm (PSM) in an anterior-toposterior sequence (Fig. 2F). Individual somites form when a block of cells at the anterior (cranial) end of the PSM undergoes mesenchymal to epithelial transition that creates a border between the newly formed somite and the PSM. Cell migration and proliferation replenish the caudal PSM as the embryo grows. This periodicity-with precisely regulated rate of somite formation and size of individual somites-is caused by the presence of the segmentation oscillator, also called the somite clock. The segmentation clock controls the periodic expression of so-called "cyclic genes," whose oscillation is synchronized between adjacent cells to form stripes of gene expression that move in posterior-to-anterior direction. The stripes cease their oscillation on reaching the anterior PSM and convey the spatio-temporal information necessary for border formation. Signaling by several pathways, including those mediated by Notch, Wnt, and fibroblast growth factor (FGF), are involved in somitogenesis, although their relative importance appears to differ among the species that have been examined (reviewed in Giudicelli and Lewis 2004; Aulehla and Pourquie 2006; Mara and Holley 2007).

Notch signaling has been suggested to participate directly in the generation of periodicity (reviewed in Giudicelli and Lewis 2004; Rida et al. 2004; Mara and Holley 2007; Shifley and Cole 2007). In zebrafish, for example, the somite clock appears to behave like a coupled negative feedback oscillator, in which Notch signaling activates the expression of downstream 
W. Zhong

transcription repressors encoded by the hairy/ $E(s p l)$ family of genes whose mRNAs translate into proteins that inhibit their own expression. This is a delayed negative feedback caused by the time required to translate mRNAs into proteins. Positive feedback is provided by specific Notch ligands, some oscillate in phase with the downstream transcription repressor genes, whereas others provide basal activation of the pathway in the PSM. Although Lfng does not appear to play a role in regulating zebrafish somite clock, Lfng transcription oscillates in the PSM in chick and mice (Forsberg et al. 1998; McGrew et al. 1998; Aulehla and Johnson 1999) and this oscillatory expression is positively regulated by Notch signaling (Barrantes et al. 1999; Morales et al. 2002). Overexpressing Lfng in the chick paraxial mesoderm abolishes the expression of cyclic genes including the endogenous $L f n g$ and leads to defects in segmentation, a phenotype similar to that caused by inhibition of Notch signaling in the PSM (Dale et al. 2003). In mice, loss of cyclic Lfng expression leads to ubiquitous Notch activation in the mutant PSM and severe segmentation defects in the thoracic and lumbar axial skeleton (primarily body formation), although cyclic Lfng expression appears to be dispensable for the more posterior (sacral and tail) skeleton (Shifley et al. 2008; Stauber et al. 2009; Oginuma et al. 2010). These findings support a model by which Lfng establishes a negative feedback loop that implements periodic inhibition of Notch through glycosylation in the Golgi, which in turn controls the rhythmic expression of cyclic genes in the posterior PSM (Fig. 2G) (Dale et al. 2003).

It is worth pointing out that a tremendous amount of knowledge has been gained in the past decades regarding how oligosaccharide moieties are added and how glycosylation modulates protein function to influence development. Developmental biologists have come to appreciate both the importance and the specificity of heparan sulphate proteoglycans, which are abundant cell-surface molecules, in modulating signaling pathways, such as those mediated by Wnt, hedgehog $(\mathrm{HH})$, FGF, and TGF family of proteins, that play essential roles in various developmental events (reviewed in
Perrimon and Bernfield 2000; Selleck 2000; Haltiwanger and Lowe 2004).

\section{GOLGI FRAGMENTATION AND RECONSTITUTION AND INTRACELLULAR SIGNALING}

Mammalian Golgi membranes are organized into interconnected stacks of flattened cisternae during interphase and are confined to the region surrounding the centrioles. Mammalian cells, however, fragment the pericentriolar Golgi stacks before entering into mitosis and subsequently disperse the Golgi fragments throughout the cytosol to ensure that both daughter cells inherit this essential organelle (reviewed in Shorter and Warren 2002; Colanzi et al. 2003; Altan-Bonnet et al. 2004). Fragmentation and dispersal of pericentriolar Golgi have been shown to be required for mammalian cells to enter into mitosis (Sutterlin et al. 2002), and studies of Numb function during mammalian neurogenesis have pointed to an interesting role for Golgi fragmentation and reconstitution in coordinating intracellular signaling and cell-cycle progression during asymmetric cell division (Zhou et al. 2007).

As discussed above, lateral inhibition and asymmetric cell division are two mechanisms that allow cells to choose different fates during development in Drosophila. Interestingly, although Notch signaling plays a central role in cell-fate specification during both processes, there are differences in how the pathway is regulated. This is exemplified by the role of the Numb protein, which affects Notch signaling during asymmetric cell division but not lateral inhibition (Petersen et al. 2006). Intrinsically asymmetric cell division is a process by which a cell produces two daughter cells that are already different at birth. Such divisions are used extensively by invertebrates to diversify cells during development. Studies using C. elegans and Drosophila show that generating two intrinsically different daughter cells requires at least three steps: a cell must first polarize itself so that one side of the cell is different from the other, followed by localizing various cell-fate determinants to only one side of the cell, and 
aligning the mitotic spindle along the axis of cell polarity so that the localized fate determinants are segregated primarily to one of the two daughter cells (reviewed in Doe 2008; Knoblich 2008; Zhong and Chia 2008). A key determinant of asymmetric cell fates in Drosophila is Numb, a cytosolic protein that is segregated asymmetrically by a wide variety of neural and nonneural precursor cells to allow the two daughter cells to adopt different fates. Because Numb specifies a variety of unrelated fates, it is believed that Numb does not specify a particular fate but simply enables the two daughter cells to choose differently between two fate options (reviewed in Jan and Jan 1995; Zhong 2003).

Genetic studies further show that Numb specifies asymmetric cell fates by inhibiting Notch to bias cell-cell communication. For example, the external sensory (ES) organ in the Drosophila periphery nervous system (PNS) consists of a neuron, a sheath cell, a hair and a socket cell. The four cells are produced by a single sensory organ precursor (SOP) cell through three rounds of asymmetric divisions (Fig. 2D). The SOP cell first divides to produce a IIA and a IIB cell. The IIA cell then divides to generate a neuron and a sheath cell, whereas the IIB divides to generate a glial cell, which either moves away or dies, and a IIIB cell, which further divides to produce a neuron and a sheath cell (Gho et al. 1999). Numb is used reiteratively by SOP cells in successive asymmetric divisions (Rhyu et al. 1994). It is symmetrically distributed during interphase but becomes localized to only one-half of the cell membrane after the SOP cell enters into mitosis and is segregated primarily into the IIB cell. This asymmetric Numb presence is essential; in the absence of Numb, both daughters adopt the IIA fate, whereas its symmetric inheritance produces two IIB cells. Although IIA and IIIB cells inherit very little Numb initially, they subsequently segregate newly synthesized Numb asymmetrically to promote hair and neuronal fates, respectively. In the SOP lineage, cell-fate transformation caused by Numb loss is identical to that caused by Notch activation in both daughter cells, whereas forcing Numb to segregate symmetrically leads to cell-fate changes that are identical to that caused by Notch loss. Because Numb and Notch double mutants show Notch mutant phenotypes, it has been suggested that Numb allows the two daughter cells to choose different fates by inhibiting Notch activity in one daughter cell to cause a bias in Notch mediated cell-cell communication, either between the two daughter cells or between the daughter cells and their environment (Guo et al. 1996; Spana and Doe 1996; Zeng et al. 1998; Lear et al. 1999). Interestingly, even though Numb is expressed by ectodermal cells during lateral inhibition, neither its loss nor its overexpression affects the specification of neural precursors like SOP cells during this process (Rhyu et al. 1994; Spana et al. 1995; Petersen et al. 2006).

How Numb regulates Notch signaling in such a context-dependent manner remains unclear. One key player in Drosophila appears to be Sanpodo, a transmembrane protein that also regulates (activates) Notch signaling specifically during asymmetric cell division (Park et al. 1998; Skeath and Doe 1998; Babaoglan et al. 2009). It has been postulated that Numb inhibits Notch signaling through Sanpodo, possibly by regulating its membrane localization (O'Connor-Giles and Skeath 2003; Hutterer and Knoblich 2005; Langevin et al. 2005; Roegiers et al. 2005; Tong et al. 2010). Sanpodo does not have an apparent vertebrate (mammalian) homolog, whereas Numb and Notch proteins are highly conserved evolutionarily. In mice, the two numb genes, m-numb (Numb) and numblike (Numbl) are functionally redundant during mouse neurogenesis and are expressed in both neural progenitor cells and neurons (Verdi et al. 1996; Zhong et al. 1996, 1997; Petersen et al. 2002). They are essential for neurogenesis but play two seemingly contradictory roles in this process. When neural progenitor cells divide asymmetrically to selfrenew and produce a neuron, Numb proteins segregate asymmetrically to promote progenitor over neuronal fates (Petersen et al. 2002, 2004). After the division, however, they are also required for the differentiation of neurons (Petersen et al. 2002; Klein et al. 2004; Huang et al. 2005). Another interesting question 
W. Zhong

regarding Numb function is whether and how the antagonistic roles of Numb and Notch are conserved during mammalian development (Petersen et al. 2006).

Unlike the process of asymmetric cell division, lateral inhibition occurs when cells are at interphase. Similarly, the two roles of mammalian Numb homologs during neurogenesis also take place in cells that differ in their cell-cycle status. Studies of the function of ACBD3, a Golgi-associated protein that can directly bind to a functionally essential Numb domain, during mouse neurogenesis point to an interesting mechanism that uses the process of Golgi fragmentation and reconstitution during cell cycle to differentially regulate Numb signaling so that the ability of Numb signaling to specify cell fates is activated only at times when the two daughter cells are being generated and deactivated shortly after they are born (Zhou et al. 2007). Like Numb and Numbl, ACBD3 is widely expressed and present in both neural progenitor cells and neurons. Unlike Numb and Numbl, which are cytosolic proteins, ACBD3 associates with the Golgi in postmitotic neurons and interphase progenitor cells. After the Golgi fragments during mitosis, however, ACBD3 is released into the cytosol. In other words, the two partners, Numb/Numbl and ACBD3, may only be able to interact with each other during mitosis, when Numb signaling is needed to distinguish the daughter cells, and the ability of Numb signaling to specify cell fates is quickly lost after the division as ACBD3 goes back to the Golgi, thereby allowing Numb to promote the differentiation of newborn neurons by tapping into a different pathway or to wait for reactivation until the next division by progenitor cells (Fig. 3). This model is supported by experiments using a myristoylated form of ACBD3, which remains in the cytosol throughout the cell cycle. The myristoylated ACBD3 can

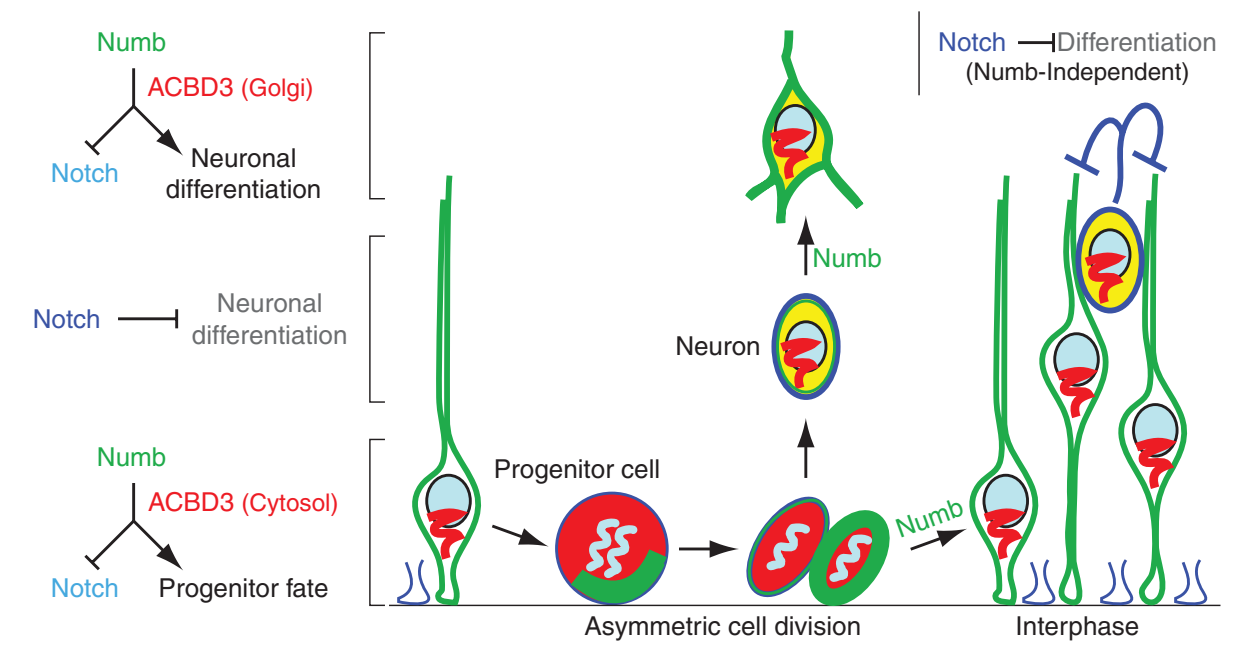

Figure 3. A model for differentially regulating Numb and Notch signaling during mammalian neurogenesis through Golgi fragmentation and reconstitution. In this model, the mammalian Numb proteins, Numb and Numbl (in green), inhibit Notch signaling to specify progenitor fates when neural progenitor cells divide asymmetrically to self-renew and produce a neuron, but this occurs only when ACBD3 (in red) is present in the cytosol after Golgi fragmentation during mitosis. Newborn neurons have higher Notch activity, which also prevents them from terminal differentiation. Newly synthesized Numb and Numbl proteins subsequently accumulate in newborn neurons but, with ACBD3 localized to the Golgi, inhibit Notch signaling by tapping into a different pathway to promote neuronal differentiation. Notch activation is also necessary for preventing progenitor cells from differentiating during interphase, and this activity is not affected by Numb presence because ACBD3 associates with the Golgi. Stem cells in other tissues may also use this mechanism to balance self-renewal and differentiation. 
enhance Numb signaling in cell-fate specification and, most importantly, its misexpression in neural progenitor cells during mouse neurogenesis inhibits neuron production by forcing both daughter cells to choose progenitor over neuronal fates.

It is unknown whether other Golgi proteins act similarly to influence intracellular signaling and development. There is an indication that abnormal Golgi fragmentation may trigger cell death, as several apoptosis signaling proteins are enriched at Golgi membranes, and fragmentation of the neuronal Golgi has been observed in several neurodegenerative diseases (reviewed in Ferri and Kroemer 2001; Gonatas et al. 2006). It is also worth noting that ACBD3 release into the cytosol likely is not simply a byproduct of Golgi fragmentation but rather a precisely regulated process. ACBD3 associates with the cytoplasmic face of Golgi membranes (Sohda et al. 2001) and remains associated with fragmented Golgi fragments (blobs) until late metaphase, when it becomes mostly cytosolic (Zhou et al. 2007). Golgi fragmentation is tightly coupled with cell-cycle progression, and the Golgi also further fragments and releases some of its contents between prometaphase and early anaphase (reviewed in Colanzi et al. 2003). Thus, it will be interesting to determine whether and how the regulators of Golgi fragmentation and reconstitution affect ACBD3 distribution. Drosophila has a highly conserved ACBD3 homolog, and the myristoylated mouse ACBD3 can inhibit Notch signaling during lateral inhibition in Drosophila (Zhou et al. 2007). The Golgi apparatus, however, exists as dispersed units in Drosophila cells and does not appear to further fragment during mitosis (Stanley et al. 1997; Kondylis et al. 2001), raising an interesting question as to whether and how this Golgibased mechanism is conserved evolutionarily.

\section{POLARIZED GOLGI DISTRIBUTION AND TRANSPORT IN NEURONAL DEVELOPMENT}

There is evidence that the Golgi also affects mammalian neurogenesis through its role in vesicle and membrane trafficking. Humans carrying mutations in the gene ADP-ribosylation factor guanine nucleotide-exchange factor-2 (ARFGEF2), which is required for vesicle and membrane trafficking from the TGN, have a small brain (microcephaly) because of defects in neural progenitor proliferation and migration (Sheen et al. 2004). Vesicle and membrane trafficking is also essential for establishing and maintaining the polarity of epithelial cells in animals. Epithelial cells serve as barriers between compartments, such as the inside and outside of the organism, and also play an essential role in transporting molecules like vital fluids and nutrients across the barrier. They are polarized into apical and basolateral plasma membranes, and the two membrane domains are structurally and functionally distinct. There is an elaborate system within the cell that allows proteins and other plasma membrane components to be specifically targeted to the apical and basolateral domains using the secretory pathway. Proteins exit the ER carrying specific signals, are sorted in the Golgi and exit from the TGN to reach their final destination through specific routes to reach apical, basolateral, and endolysosomal domains (reviewed in Rodriguez-Boulan et al. 2005).

Neuronal morphogenesis exemplifies how cells use the apical and basolateral sorting routes differently to meet their specific functional needs. Neurons represent an extreme case of cellular polarization. Neuronal development involves outgrowth of unique cellular processes (neurites) that develop into two distinct compartments, axons and dendrites, that are essential for building the neural network. A typical neuron has multiple dendrites to receive signals and a single, often much longer axon to relay the information within the neural circuit. Dendrites have elaborate arbors and stereotyped patterns of branching, whereas axons are capable of traversing extremely long distances, some nearly the entire length of the animal body. Because of the presence of axons and dendrites, the surface area of a neuron can easily reach millions of square micrometers compared to just a few thousand square micrometers for average animal cells, and the secretory pathway is essential for delivering proteins and other components that drive the 
W. Zhong

enormous expansion of plasma membrane during neuronal growth as well as its maintenance (reviewed in Kennedy and Ehlers 2006; Pfenninger 2009; Tahirovic and Bradke 2009; Jan and Jan 2010). In addition to functional and morphological differences, axons and dendrites also differ in their molecular composition and the timing, rate, and dynamics of their growth. One hypothesis is that neurons and polarized epithelial cells may share a common mechanism for protein targeting, with apical equivalent to axonal and basolateral equivalent to somatodendritic (Dotti and Simons 1990). Although many proteins indeed are targeted in neurons and epithelial cells like the MDCK cells as this model predicts, the spatial organization of secretory pathway components, particularly the Golgi, is distinct in neurons, which allows the secretory pathway to play a rather unexpected role in establishing and maintaining the polarity of neurons (reviewed in Hanus and Ehlers 2008).

In most mammalian cells, the ER is dispersed throughout the cytoplasm, whereas the Golgi membranes are organized into a single unit confined to the region surrounding the centrioles. The ER in neurons is also distributed throughout the cytoplasm. Neuronal Golgi, however, is not only organized into the pericentriolar membrane array seen in other cells but also can be found as discrete structures_-called "Golgi outposts" - that are dispersed in dendrites and excluded from axons (Gardiol et al. 1999; Pierce et al. 2001; Horton and Ehlers 2003; Aridor et al. 2004). Pyramidal neurons from the mammalian hippocampus have multiple dendrites that differ in length and complexity. Studies by Ehlers and colleagues revealed that Golgi outposts are not present in all dendrites but instead are selectively partitioned into the longest dendrite and concentrate at branch points. Equally important, the Golgi apparatus within the cell body also orients toward the longest dendrite. Their studies further show this polarized Golgi distribution occurs before neurites show apparent differences, and post-Golgi secretory trafficking in these neurons is directed predominantly toward the longest dendrite, suggesting this unexpected spatial organization of neuronal secretory trafficking is important for asymmetric dendritic growth and morphogenesis. Indeed, blocking forward secretory trafficking using brefeldin A, a drug that reversibly disassembles the Golgi, or a kinase-dead mutant of protein Kinase D1, which prevents cargo budding from the TGN, severely affects dendrite growth in young neurons and the maintenance of dendrites in more mature neurons. More importantly, disruption of neuronal Golgi organization during asymmetric dendrite growth by overexpressing the Golgi structural protein GRASP65, which causes Golgi vesiculation and dispersal into multiple dendrites but does not affect secretory function per se, produces neurons with symmetric neurites without affecting total dendrite outgrowth (Horton et al. 2005). Taken together, these findings provide compelling evidence that the Golgi not only is important in driving membrane expansion during neuronal growth but also plays a highly specific role in establishing and maintaining neuronal polarity.

The role of polarized Golgi distribution and transport in neuronal morphogenesis is also evolutionarily conserved. Jan and colleagues used forward genetic screens in Drosophila to isolate mutants defective in dendrite development focusing on a class of neurons, class IV da neurons, that are morphologically similar to mammalian neurons (reviewed in Jan and Jan 2010). Mutants of one class, dendritic arbor reduction (dar), affect dendrite (but not axon) development, and the genes affected in three such mutants, dar2, dar3, and dar6, respectively, are Drosophila homologs of Sec23, Sar1, and $R a b 1$, which play essential roles in ER-to-Golgi transport mediated by the COPII vesicles (Ye et al. 2007). In an elegant set of experiments focusing on dar3/Sar1, Ye et al. showed that the gene acts cell autonomously to influence dendrite growth in Drosophila and knocking down Sar1 in mammalian hippocampal neurons to block ER-to-Golgi transport similarly reduces dendrite growth without affecting axon growth. The Golgi exists as dispersed units throughout the cytoplasm in Drosophila class IV da neurons. These neurons also have Golgi outposts that are present in both major and fine 
dendritic branches but rarely in axons, and these outposts contribute to dendrite growth. These findings show that, in addition to its ubiquitous importance in plasma membrane generation, the ER-to-Golgi trafficking can also play a highly specific role in promoting dendrite, but not axon, development partly through polarized distribution of some of the components of the secretory pathway, including Golgi outposts.

Manipulating the activity of other regulators of Golgi dynamics and membrane trafficking, including protein kinase D (PKD), Syntaxin 16 , and secretory pathway $\mathrm{Ca}^{2+}$ ATPase isoform 1 (SPCA) also affects neuronal polarity (Bisbal et al. 2008; Chua and Tang 2008; Yin et al. 2008; Czondor et al. 2009; Sepulveda et al. 2009). It is worth noting the role played by the Golgi likely needs to be viewed in the context of the entire secretory pathway. For example, recycling endosomes also have a central role in establishing and maintaining epithelial polarity by affecting intracellular sorting and polarized trafficking of apical and basolateral membrane components (reviewed in Golachowska et al. 2010). During neuronal morphogenesis, one can speculate that the reason for defects in ER-to-Golgi trafficking to differentially affect dendrite, but not axon, outgrowth is in part caused by differences between axons and dendrites in the levels of endocytosis and where the recycled membrane components are delivered to (Fig. 4).

\section{CONCLUDING REMARKS}

Highlighted in this article are examples of how the Golgi is used in a highly context-dependent manner to influence pathways important in invertebrate and vertebrate development. Interestingly, although the role of the Golgi as a venue for protein modification and transport is evolutionarily conserved, different species and different cell types appear to use this role differently to influence cellular differentiation. For example, Rhomboid proteins, which spatially and temporally confine EGFR signaling during Drosophila development by regulating ligand transport and processing in the ER and the Golgi of signaling cells, apparently are not used by mammals to control EGFR signaling (reviewed in Freeman 2004; Urban 2006). There is also evidence that protein-specific cleavage and glycosylation occur at distinct sites within the Golgi. In Drosophila, the Golgi units harboring Rhomboid appear to be distinct from those enzymes involved in protein glycosylation (Yano et al. 2005). Equally interesting, different

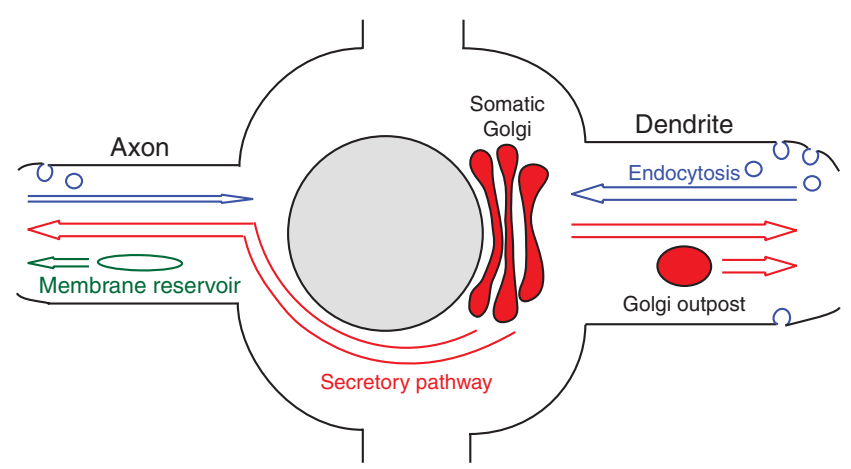

Figure 4. A model for how inhibition of the secretory pathway affects dendrite, but not axon, growth during neuronal morphogenesis. Because dendrites may have higher levels of endocytosis and most vesicles deliver the recycled membrane components to the soma through retrograde transport, they are more dependent on membrane supplies delivered through the secretory pathway, including those by the Golgi outposts. Axons, on the other hand, are less dependent on the secretory pathway because they may have lower levels of endocytosis, a larger membrane reservoir, and more efficient transport of post-Golgi vesicles (based on a sketch courtesy of Bing Ye, University of Michigan). 
W. Zhong

species and different cell types organize the Golgi apparatus differently and exploit the differences to influence cellular differentiation and morphogenesis. The mammalian ACBD3 protein appears to regulate the timing of cellfate determination during mammalian neurogenesis by taking advantage of the process of Golgi fragmentation and reconstitution (Zhou et al. 2007). Golgi membranes, however, exist as dispersed units in Drosophila cells and do not undergo further fragmentation during cell cycle (Stanley et al. 1997; Kondylis et al. 2001). On the other hand, neurons in both Drosophila and mammals have unique Golgi outposts that are not found in other cell types, and such outposts are used to promote dendrite, but not axon, outgrowth during neuronal morphogenesis (Horton et al. 2005; Ye et al. 2007). In other words, because of the diverse roles played by the Golgi in different species and different cell types, our understanding of how the Golgi influences development likely is at a very early stage and future studies likely will yield fascinating mechanisms involving the Golgi in regulating essential developmental pathways.

\section{ACKNOWLEDGMENTS}

I thank Dr. Scott Holley and Dr. Bing Ye for figures and discussions. I also apologize to colleagues whose publications are not cited here because of the narrow focus of this article. I am grateful for the support from the National Institutes of Health (NINDS and NICHD), Susan G. Komen for the Cure and Alex's Lemonade Stand Foundation for Childhood Cancer, which has made the work in my laboratory possible.

\section{REFERENCES}

Altan-Bonnet N, Sougrat R, Lippincott-Schwartz J. 2004. Molecular basis for Golgi maintenance and biogenesis. Curr Opin Cell Biol 16: 364-372.

Aridor M, Guzik AK, Bielli A, Fish KN. 2004. Endoplasmic reticulum export site formation and function in dendrites. J Neurosci 24: 3770-3776.

Aulehla A, Johnson RL 1999. Dynamic expression of lunatic fringe suggests a link between notch signaling and an autonomous cellular oscillator driving somite segmentation. Dev Biol 207: 49-61.
Aulehla A, Pourquie O. 2006. On periodicity and directionality of somitogenesis. Anat Embryol 211 (Suppl 1): 3-8.

Babaoglan AB, O'Connor-Giles KM, Mistry H, Schickedanz A, Wilson BA, Skeath JB. 2009. Sanpodo: A contextdependent activator and inhibitor of Notch signaling during asymmetric divisions. Development 136: 40894098.

Barrantes IB, Elia AJ, Wunsch K, Hrabe de Angelis MH, Mak TW, Rossant J, Conlon RA, Gossler A, de la Pompa JL. 1999. Interaction between Notch signalling and Lunatic fringe during somite boundary formation in the mouse. Curr Biol 9: 470-480.

Bisbal M, Conde C, Donoso M, Bollati F, Sesma J, Quiroga S, Diaz Anel A, Malhotra V, Marzolo MP, Caceres A. 2008. Protein kinase $d$ regulates trafficking of dendritic membrane proteins in developing neurons. J Neurosci 28: 9297-9308.

Blaumueller CM, Qi H, Zagouras P, Artavanis-Tsakonas S. 1997. Intracellular cleavage of Notch leads to a heterodimeric receptor on the plasma membrane. Cell 90: 281-291.

Bogda S, Klambt C. 2001. Epidermal growth factor receptor signaling. Curr Biol 11: R292-R295.

Bray SJ. 2006. Notch signalling: A simple pathway becomes complex. Nat Rev Mol Cell Biol 7: 678-689.

Bruckner K, Perez L, Clausen H, Cohen S. 2000. Glycosyltransferase activity of Fringe modulates Notch- $\delta$ interactions. Nature 406: 411-415.

Chua CE, Tang BL. 2008. Syntaxin 16 is enriched in neuronal dendrites and may have a role in neurite outgrowth. Mol Membr Biol 25: 35-45.

Colanzi A, Suetterlin C, Malhotra V. 2003. Cell-cycle-specific Golgi fragmentation: How and why? Curr Opin Cell Biol 15: 462-467.

Czondor K, Ellwanger K, Fuchs YF, Lutz S, Gulyas M, Mansuy IM, Hausser A, Pfizenmaier K, Schlett K. 2009. Protein kinase D controls the integrity of Golgi apparatus and the maintenance of dendritic arborization in hippocampal neurons. Mol Biol Cell 20: 2108-2120.

Dale JK, Maroto M, Dequeant ML, Malapert P, McGrew M, Pourquie O. 2003. Periodic notch inhibition by lunatic fringe underlies the chick segmentation clock. Nature 421: $275-278$.

Doe CQ. 2008. Neural stem cells: Balancing self-renewal with differentiation. Development 135: 1575-1587.

Dotti CG, Simons K. 1990. Polarized sorting of viral glycoproteins to the axon and dendrites of hippocampal neurons in culture. Cell 62: 63-72.

Evrard YA, Lun Y, Aulehla A, Gan L, Johnson RL. 1998. lunatic fringe is an essential mediator of somite segmentation and patterning. Nature 394: 377-381.

Ferri KF, Kroemer G. 2001. Organelle-specific initiation of cell death pathways. Nat Cell Biol 3: E255-E263.

Fleming RJ, Gu Y, Hukriede NA. 1997. Serrate-mediated activation of Notch is specifically blocked by the product of the gene fringe in the dorsal compartment of the Drosophila wing imaginal disc. Development 124: 2973-2981.

Forsberg H, Crozet F, Brown NA. 1998. Waves of mouse Lunatic fringe expression, in four-hour cycles at 
two-hour intervals, precede somite boundary formation. Curr Biol 8: 1027-1030.

Freeman M. 1996. Reiterative use of the EGF receptor triggers differentiation of all cell types in the Drosophila eye. Cell 87: 651-660.

Freeman M. 2004. Proteolysis within the membrane: Rhomboids revealed. Nat Rev Mol Cell Biol 5: 188-197.

Gabay L, Seger R, Shilo BZ. 1997. In situ activation pattern of Drosophila EGF receptor pathway during development. Science 277: 1103-1106.

Gardiol A, Racca C, Triller A. 1999. Dendritic and postsynaptic protein synthetic machinery. J Neurosci 19: $168-179$.

Gho M, Bellaiche Y, Schweisguth F. 1999. Revisiting the Drosophila microchaete lineage: A novel intrinsically asymmetric cell division generates a glial cell. Development 126: 3573-3584.

Giudicelli F, Lewis J. 2004. The vertebrate segmentation clock. Curr Opin Genet Dev 14: 407-414.

Golachowska MR, Hoekstra D, van Ijzendoorn SC. 2010. Recycling endosomes in apical plasma membrane domain formation and epithelial cell polarity. Trends Cell Biol 20: 618-626.

Golembo M, Raz E, Shilo BZ. 1996. The Drosophila embryonic midline is the site of Spitz processing, and induces activation of the EGF receptor in the ventral ectoderm. Development 122: 3363-3370.

Gonatas NK, Stieber A, Gonatas JO. 2006. Fragmentation of the Golgi apparatus in neurodegenerative diseases and cell death. J Neurol Sci 246: 21-30.

Guichard A, Biehs B, Sturtevant MA, Wickline L, Chacko J, Howard K, Bier E. 1999. Rhomboid and Star interact synergistically to promote EGFR/MAPK signaling during Drosophila wing vein development. Development 126: 2663-2676.

Guo M, Jan LY, Jan YN. 1996. Control of daughter cell fates during asymmetric division: Interaction of Numb and Notch. Neuron 17: 27-41.

Haines N, Irvine KD. 2003. Glycosylation regulates Notch signalling. Nat Rev Mol Cell Biol 4: 786-797.

Haltiwanger RS, Lowe JB. 2004. Role of glycosylation in development. Annu Rev Biochem 73: 491-537.

Hanus C, Ehlers MD. 2008. Secretory outposts for the local processing of membrane cargo in neuronal dendrites. Traffic 9: 1437-1445.

Heberlein U, Rubin GM. 1991. Star is required in a subset of photoreceptor cells in the developing Drosophila retina and displays dosage sensitive interactions with rough. Dev Biol 144: 353-361.

Heberlein U, Hariharan IK, Rubin GM. 1993. Star is required for neuronal differentiation in the Drosophila retina and displays dosage-sensitive interactions with Ras1. Dev Biol 160: 51-63.

Horton AC, Ehlers MD. 2003. Dual modes of endoplasmic reticulum-to-Golgi transport in dendrites revealed by live-cell imaging. J Neurosci 23: 6188-6199.

Horton AC, Racz B, Monson EE, Lin AL, Weinberg RJ Ehlers MD. 2005. Polarized secretory trafficking directs cargo for asymmetric dendrite growth and morphogenesis. Neuron 48: 757-771.
Huang EJ, Li H, Tang AA, Wiggins AK, Neve RL, Zhong W Jan LY, Jan YN. 2005. Targeted deletion of numb and numblike in sensory neurons reveals their essential functions in axon arborization. Genes Dev 19: 138-151.

Hutterer A, Knoblich JA. 2005. Numb and $\alpha$-Adaptin regulate Sanpodo endocytosis to specify cell fate in Drosophila external sensory organs. EMBO Rep 6: 836-842.

Irvine KD, Wieschaus E. 1994. FFringe, a boundary-specific signaling molecule, mediates interactions between dorsal and ventral cells during Drosophila wing development. Cell 79: 595-606.

Jafar-Nejad H, Leonardi J, Fernandez-Valdivia R. 2010. Role of glycans and glycosyltransferases in the regulation of Notch signaling. Glycobiology 20: 931-949.

Jan YN, Jan LY. 1995. Maggot's hair and bug's eye: Role of cell interactions and intrinsic factors in cell fate specification. Neuron 14: 1-5.

Jan YN, Jan LY. 2010. Branching out: mechanisms of dendritic arborization. Nat Rev Neurosci 11: 316-328.

Kennedy MJ, Ehlers MD. 2006. Organelles and trafficking machinery for postsynaptic plasticity. Ann Rev Neurosci 29: 325-362.

Kim J, Irvine KD, Carroll SB. 1995. Cell recognition, signal induction, and symmetrical gene activation at the dorsalventral boundary of the developing Drosophila wing. Cell 82: $795-802$.

Klein T, Arias AM. 1998. Interactions among $\delta$, Serrate and Fringe modulate Notch activity during Drosophila wing development. Development 125: 2951-2962.

Klein AL, Zilian O, Suter U, Taylor V. 2004. Murine numb regulates granule cell maturation in the cerebellum. Dev Biol 266: 161-177.

Knoblich JA. 2008. Mechanisms of asymmetric stem cell division. Cell 132: 583-597.

Kondylis V, Goulding SE, Dunne JC, Rabouille C. 2001. Biogenesis of Golgi stacks in imaginal discs of Drosophila melanogaster. Mol Biol Cell 12: 2308-2327.

Lake RJ, Grimm LM, Veraksa A, Banos A, Artavanis-Tsakonas S. 2009. In vivo analysis of the Notch receptor S1 cleavage. PloS One 4: e6728.

Langevin J, Le Borgne R, Rosenfeld F, Gho M, Schweisguth F, Bellaiche Y. 2005. Lethal giant larvae controls the localization of notch-signaling regulators numb, neuralized, and Sanpodo in Drosophila sensory-organ precursor cells. Curr Biol 15: 955-962.

Lear BC, Skeath JB, Patel NH. 1999. Neural cell fate in rcal and cycA mutants: The roles of intrinsic and extrinsic factors in asymmetric division in the Drosophila central nervous system. Mech Dev 88: 207-219.

Lee JR, Urban S, Garvey CF, Freeman M. 2001. Regulated intracellular ligand transport and proteolysis control EGF signal activation in Drosophila. Cell 107: 161-171.

Lee MC, Miller EA, Goldberg J, Orci L, Schekman R. 2004. Bi-directional protein transport between the ER and Golgi. Annu Rev Cell Dev Biol 20: 87-123.

Logeat F, Bessia C, Brou C, LeBail O, Jarriault S, Seidah NG, Israel A. 1998. The Notch1 receptor is cleaved constitutively by a furin-like convertase. Proc Natl Acad Sci 95: $8108-8112$. 
W. Zhong

Louvi A, Artavanis-Tsakonas S. 2006. Notch signalling in vertebrate neural development. Nat Rev Neurosci 7: 93-102.

Mara A, Holley SA. 2007. Oscillators and the emergence of tissue organization during zebrafish somitogenesis. Trends Cell Biol 17: 593-599.

McGrew MJ, Dale JK, Fraboulet S, Pourquie O. 1998. The lunatic fringe gene is a target of the molecular clock linked to somite segmentation in avian embryos. Curr Biol 8: 979-982.

Moloney DJ, Panin VM, Johnston SH, Chen J, Shao L, Wilson R, Wang Y, Stanley P, Irvine KD, Haltiwanger RS, et al. 2000. Fringe is a glycosyltransferase that modifies Notch. Nature 406: 369-375.

Morales AV, Yasuda Y, Ish-Horowicz D. 2002. Periodic Lunatic fringe expression is controlled during segmentation by a cyclic transcriptional enhancer responsive to notch signaling. Dev Cell 3: 63-74.

Mumm JS, Kopan R. 2000. Notch signaling: From the outside in. Dev Biol 228: 151-165.

Munro S, Freeman M. 2000. The notch signalling regulator fringe acts in the Golgi apparatus and requires the glycosyltransferase signature motif DXD. Curr Biol 10: 813-820.

O'Connor-Giles KM, Skeath JB. 2003. Numb inhibits membrane localization of Sanpodo, a four-pass transmembrane protein, to promote asymmetric divisions in Drosophila. Dev Cell 5: 231-243.

Oginuma M, Takahashi Y, Kitajima S, Kiso M, Kanno J, Kimura A, Saga Y. 2010. The oscillation of Notch activation, but not its boundary, is required for somite border formation and rostral-caudal patterning within a somite. Development 137: 1515-1522.

Okajima T, Irvine KD. 2002. Regulation of notch signaling by o-linked fucose. Cell 111: 893-904.

Panin VM, Papayannopoulos V, Wilson R, Irvine KD. 1997. Fringe modulates Notch-ligand interactions. Nature 387: 908-912.

Park M, Yaich LE, Bodmer R. 1998. Mesodermal cell fate decisions in Drosophila are under the control of the lineage genes numb, Notch, and sanpodo. Mech Dev 75: $117-126$.

Perrimon N, Bernfield M. 2000. Specificities of heparan sulphate proteoglycans in developmental processes. Nature 404: 725-728.

Petersen PH, Zou K, Hwang JK, Jan YN, Zhong W. 2002. Progenitor cell maintenance requires numb and numblike during mouse neurogenesis. Nature 419: 929-934.

Petersen PH, Zou K, Krauss S, Zhong W. 2004. Continuing role for mouse Numb and Numbl in maintaining progenitor cells during cortical neurogenesis. Nat Neurosci 7: 803-811.

Petersen PH, Tang H, Zou K, Zhong W. 2006. The enigma of the Numb-Notch relationship during mammalian embryogenesis. Dev Neurosci 28: 156-168.

Pfenninger KH. 2009. Plasma membrane expansion: A neuron's Herculean task. Nat Rev Neurosci 10: 251-261.

Pierce JP, Mayer T, McCarthy JB. 2001. Evidence for a satellite secretory pathway in neuronal dendritic spines. Curr Biol 11: 351-355.
Price JV, Clifford RJ, Schupbach T. 1989. The maternal ventralizing locus torpedo is allelic to faint little ball, an embryonic lethal, and encodes the Drosophila EGF receptor homolog. Cell 56: 1085-1092.

Rauskolb C, Correia T, Irvine KD. 1999. Fringe-dependent separation of dorsal and ventral cells in the Drosophila wing. Nature 401: 476-480.

Rhyu MS, Jan LY, Jan YN. 1994. Asymmetric distribution of numb protein during division of the sensory organ precursor cell confers distinct fates to daughter cells. Cell 76: 477-491.

Rida PC, Le Minh N, Jiang YJ. 2004. A Notch feeling of somite segmentation and beyond. Dev Biol 265: 2-22.

Rodriguez-Boulan E, Kreitzer G, Musch A. 2005. Organization of vesicular trafficking in epithelia. Nat Rev Mol Cell Biol 6: 233-247.

Roegiers F, Jan LY, Jan YN. 2005. Regulation of membrane localization of Sanpodo by lethal giant larvae and neuralized in asymmetrically dividing cells of Drosophila sensory organs. Mol Biol Cell 16: 3480-3487.

Rousso T, Lynch J, Yogev S, Roth S, Schejter ED, Shilo BZ. 2010. Generation of distinct signaling modes via diversification of the Egfr ligand-processing cassette. Development 137: 3427-3437.

Rutledge BJ, Zhang K, Bier E, Jan YN, Perrimon N. 1992. The Drosophila spitz gene encodes a putative EGF-like growth factor involved in dorsal-ventral axis formation and neurogenesis. Genes Dev 6: 1503-1517.

Schejter ED, Shilo BZ. 1989. The Drosophila EGF receptor homolog (DER) gene is allelic to faint little ball, a locus essential for embryonic development. Cell 56: $1093-$ 1104.

Schweitzer R, Shaharabany M, Seger R, Shilo BZ. 1995. Secreted Spitz triggers the DER signaling pathway and is a limiting component in embryonic ventral ectoderm determination. Genes Dev 9: 1518-1529.

Selleck SB. 2000. Proteoglycans and pattern formation: Sugar biochemistry meets developmental genetics. Trends Genet 16: 206-212.

Sepulveda MR, Vanoevelen J, Raeymaekers L, Mata AM, Wuytack F. 2009. Silencing the SPCA1 (secretory pathway $\mathrm{Ca}^{2+}$-ATPase isoform 1) impairs $\mathrm{Ca}^{2+}$ homeostasis in the Golgi and disturbs neural polarity. J Neurosci 29: 12174-12182.

Sheen VL, Ganesh VS, Topcu M, Sebire G, Bodell A, Hill RS, Grant PE, Shugart YY, Imitola J, Khoury SJ, et al. 2004. Mutations in ARFGEF2 implicate vesicle trafficking in neural progenitor proliferation and migration in the human cerebral cortex. Nat Genet 36: 69-76.

Shi S, Stanley P. 2003. Protein O-fucosyltransferase 1 is an essential component of Notch signaling pathways. Proc Natl Acad Sci 100: 5234-5239.

Shifley ET, Cole SE. 2007. The vertebrate segmentation clock and its role in skeletal birth defects. Birth Def Res C 81: 121-133.

Shifley ET, Vanhorn KM, Perez-Balaguer A, Franklin JD, Weinstein M, Cole SE. 2008. Oscillatory lunatic fringe activity is crucial for segmentation of the anterior but not posterior skeleton. Development 135: 899-908.

Shorter J, Warren G. 2002. Golgi architecture and inheritance. Annu Rev Cell Dev Biol 18: 379-420. 
Skeath JB, Doe CQ. 1998. Sanpodo and Notch act in opposition to Numb to distinguish sibling neuron fates in the Drosophila CNS. Development 125: 1857-1865.

Sohda M, Misumi Y, Yamamoto A, Yano A, Nakamura N, Ikehara Y. 2001. Identification and characterization of a novel Golgi protein, GCP60, that interacts with the integral membrane protein giantin. J Biol Chem 276: 45298-45306.

Spana EP, Doe CQ. 1996. Numb antagonizes Notch signaling to specify sibling neuron cell fates. Neuron 17: 21-26.

Spana EP, Kopczynski C, Goodman CS, Doe CQ. 1995. Asymmetric localization of numb autonomously determines sibling neuron identity in the Drosophila CNS. Development 121: 3489-3494.

Stanley P. 2007. Regulation of Notch signaling by glycosylation. Curr Opin Struct Biol 17: 530-535.

Stanley H, Botas J, Malhotra V. 1997. The mechanism of Golgi segregation during mitosis is cell type-specific. Proc Natl Acad Sci 94: 14467-14470.

Stauber M, Sachidanandan C, Morgenstern C, Ish-Horowicz D. 2009. Differential axial requirements for lunatic fringe and Hes7 transcription during mouse somitogenesis. PloS One 4: e7996.

Sutterlin C, Hsu P, Mallabiabarrena A, Malhotra V. 2002. Fragmentation and dispersal of the pericentriolar Golgi complex is required for entry into mitosis in mammalian cells. Cell 109: 359-369.

Tahirovic S, Bradke F. 2009. Neuronal polarity. Cold Spring Harb Perspect Biol 1: a001644.

Takeuchi H, Haltiwanger RS. 2010. Role of glycosylation of Notch in development. Semin Cell Dev Biol 21: 638-645.

Tien AC, Rajan A, Bellen HJ. 2009. A Notch updated. J Cell Biol 184: 621-629.

Tong X, Zitserman D, Serebriiskii I, Andrake M, Dunbrack R, Roegiers F. 2010. Numb independently antagonizes Sanpodo membrane targeting and Notch signaling in Drosophila sensory organ precursor cells. Mol Biol Cell 21: $802-810$.

Tsruya R, Schlesinger A, Reich A, Gabay L, Sapir A, Shilo BZ. 2002. Intracellular trafficking by Star regulates cleavage of the Drosophila EGF receptor ligand Spitz. Genes Dev 16: 222-234.

Tsruya R, Wojtalla A, Carmon S, Yogev S, Reich A, Bibi E, Merdes G, Schejter E, Shilo BZ. 2007. Rhomboid cleaves Star to regulate the levels of secreted Spitz. EMBO J 26: 1211-1220.

Urban S. 2006. Rhomboid proteins: Conserved membrane proteases with divergent biological functions. Genes Dev 20: 3054-3068.
Urban S, Lee JR, Freeman M. 2001. Drosophila rhomboid-1 defines a family of putative intramembrane serine proteases. Cell 107: 173-182.

Verdi JM, Schmandt R, Bashirullah A, Jacob S, Salvino R, Craig CG, Program AE, Lipshitz HD, McGlade CJ. 1996. Mammalian NUMB is an evolutionarily conserved signaling adapter protein that specifies cell fate. Curr Biol 6: $1134-1145$.

Wasserman JD, Urban S, Freeman M. 2000. A family of rhomboid-like genes: Drosophila rhomboid-1 and roughoid/rhomboid-3 cooperate to activate EGF receptor signaling. Genes Dev 14: 1651-1663.

Yano H, Yamamoto-Hino M, Abe M, Kuwahara R, Haraguchi S, Kusaka I, Awano W, Kinoshita-Toyoda A, Toyoda H, Goto S. 2005. Distinct functional units of the Golgi complex in Drosophila cells. Proc Natl Acad Sci 102: 13467-13472.

Ye B, Zhang Y, Song W, Younger SH, Jan LY, Jan YN. 2007. Growing dendrites and axons differ in their reliance on the secretory pathway. Cell 130: 717-729.

Yin DM, Huang YH, Zhu YB, Wang Y. 2008. Both the establishment and maintenance of neuronal polarity require the activity of protein kinase D in the Golgi apparatus. J Neurosci 28: 8832-8843.

Yogev S, Schejter ED, Shilo BZ. 2008. Drosophila EGFR signalling is modulated by differential compartmentalization of Rhomboid intramembrane proteases. $E M B O J$ 27: 1219-1230.

Zeng C, Younger-Shepherd S, Jan LY, Jan YN. 1998. Delta and Serrate are redundant Notch ligands required for asymmetric cell divisions within the Drosophila sensory organ lineage. Genes Dev 12: 1086-1091.

Zhang N, Gridley T. 1998. Defects in somite formation in lunatic fringe-deficient mice. Nature 394: 374-377.

Zhong W. 2003. Diversifying neural cells through order of birth and asymmetry of division. Neuron 37: 11-14.

Zhong W, Chia W. 2008. Neurogenesis and asymmetric cell division. Curr Opin Neurobiol 18: 4-11.

Zhong W, Feder JN, Jiang MM, Jan LY, Jan YN. 1996. Asymmetric localization of a mammalian numb homolog during mouse cortical neurogenesis. Neuron 17: 43-53.

Zhong W, Jiang MM, Weinmaster G, Jan LY, Jan YN. 1997. Differential expression of mammalian Numb, Numblike and Notch1 suggests distinct roles during mouse cortical neurogenesis. Development 124: 1887-1897.

Zhou Y, Atkins JB, Rompani SB, Bancescu DL, Petersen PH, Tang H, Zou K, Stewart SB, Zhong W. 2007. The mammalian Golgi regulates numb signaling in asymmetric cell division by releasing ACBD3 during mitosis. Cell 129: $163-178$. 


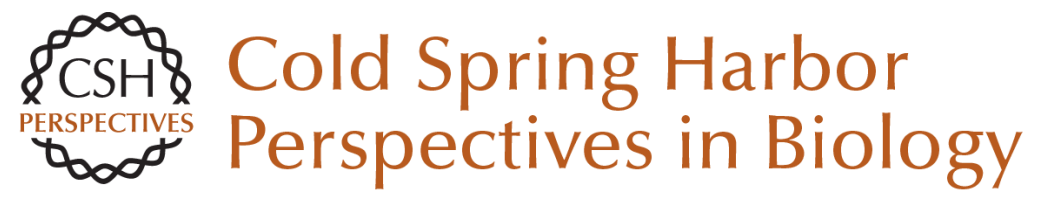

\section{Golgi during Development}

Weimin Zhong

Cold Spring Harb Perspect Biol 2011; doi: 10.1101/cshperspect.a005363 originally published online July 18, 2011

\section{Subject Collection The Golgi}

Structure of Golgi Transport Proteins

Daniel Kümmel and Karin M. Reinisch

\section{Golgi Biogenesis}

Yanzhuang Wang and Joachim Seemann

Golgi Glycosylation and Human Inherited

Diseases

Hudson H. Freeze and Bobby G. Ng

Models for Golgi Traffic: A Critical Assessment Benjamin S. Glick and Alberto Luini

\section{Architecture of the Mammalian Golgi} Judith Klumperman

Evolution and Diversity of the Golgi Mary J. Klute, Paul Melançon and Joel B. Dacks

Evolutionary Forces Shaping the Golgi

Glycosylation Machinery: Why Cell Surface

Glycans Are Universal to Living Cells Ajit Varki

Golgi Positioning

Smita Yadav and Adam D. Linstedt
Golgi and Related Vesicle Proteomics: Simplify to Identify Joan Gannon, John J.M. Bergeron and Tommy Nilsson

Organization of SNAREs within the Golgi Stack Jörg Malsam and Thomas H. Söllner

Golgi during Development Weimin Zhong

Entry and Exit Mechanisms at the cis-Face of the Golgi Complex Andrés Lorente-Rodríguez and Charles Barlowe

COPI Budding within the Golgi Stack Vincent Popoff, Frank Adolf, Britta Brügger, et al.

Mechanisms of Protein Retention in the Golgi David K. Banfield

The Golgin Coiled-Coil Proteins of the Golgi

Apparatus Sean Munro

Signaling at the Golgi Peter Mayinger

For additional articles in this collection, see http://cshperspectives.cshlp.org/cgi/collection/

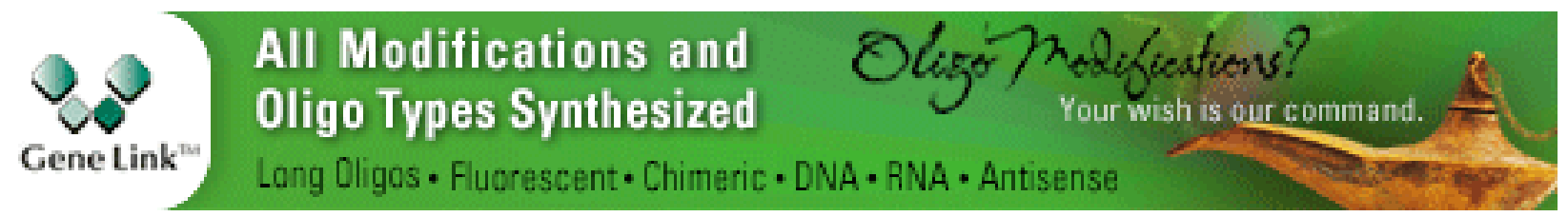

Copyright @ 2011 Cold Spring Harbor Laboratory Press; all rights reserved 